\title{
Biophysics and Nanotechnologies: Problems and Prospects
}

DOI: $10.1134 / \mathrm{S} 0006350911050265$

In 2009 and 2010, the RAS Scientific Council on Biological Physics held plenary sessions devoted to discussing the current state of biophysical problems in the light of development of nanotechnological approaches.

Modern nanotechnologies mark the new revolutionary step of scientific and technical development in the XXI century. This field of natural science and technological progress bears a universal interdisciplinary character. In the framework of this direction, nanostructures, nanomaterials and nanodevices are created, processes taking place on the nanometer scale are studied. Approaches are developed for controlling separate atoms, molecules and supramolecular formations. Novel materials acquire unique physical, chemical and biological properties; particular achievements are so-called defect-free materials distinguished by outstanding strength, and materials with high conductivity, as well as nanomaterials promising for sliding couples. Many merits of nanotechnologies are due simply to the effect of miniaturization. The energy and material expenditures are radically reduced by orders of magnitude; accordingly, the output of devices can be raised.

In addition to molecular design long mastered by chemists, nanotechnologies allow synthesis of novel molecules in strongly inhomogeneous electromagnetic fields. An adjacent topic is design of catalysts with nanostructures for reactions of selective catalysis as a particular case of heterogeneous catalysis.

With the ongoing microminiaturization of computer parts, perhaps the next generation will master the nanometer scale for conductors, transistors, rectifiers and acoustic elements. Especially promising is the vigorously developing field that may be called nanoinstrument design, including construction of scanning tunnel and atomic force microscopes, multitip systems based on nanolasers, miniature supersensitive pickups, nanorobots, and reaching down to the subatomic level, with particle nanoaccelerators for controlled non-statistical nuclear reactions.

Nanotechnologies are at the leading edge of scientific and technical development, are synonymous with innovation and progress. On the other hand, in fact the nanoworld coexists with the humankind throughout its history. And nanotechnologies have been known to the humankind for millennia as biotechnologies. These are first of all the technologies connected with the very human existence, including food chains and medicine.

Biology opens new prospects in nanotechnologies and horizons of the use of nanodevices, inasmuch as it is believed that over the millions of years of evolution these molecular biological devices have reached perfection. On the other side, in nanotechnologies one may create unique molecular model where structures are built on biological principles on organization with non-biological materials. Also important is replacement and reproduction of biological functions with the aid of technical means.

The contribution of modern biophysics to development of nanobiotechnologies is not restricted to striking examples of bionanomechanisms. It provides a theoretical basis for understanding the fundamental physical regularities acting in nanobiosystems. The theoretical approach has recently been joined by the possibility of unique experiments. It becomes possible not only to calculate the parameters of microsystems with accuracy heretofore accessible only to microscopic or selective methods, but also to measure them directly.

The goal of nanotechnology as applied to biophysics is creation of nanosized biotechnical devices that would, like natural cellular systems, destroy transformed cells, first recognizing them. At that these device must possess a function controlling the formation of corresponding proteomic complexes.

The system of in vivo nanotechnologies encompasses all levels of organization of bioobjects, starting with molecules, their complexes and ending with the cell population level. Such biophysical systems are photosynthesis, processes of energy production, transmission and transformation (including generation of electricity), protein biosynthesis, processes of regulation of gene expression.

Biophysics gives numerous examples of hierarchical organization of both structures and functions. In particular, it becomes clear how to ensure an entropic advantage of self-assembly by using different degrees of polymerization. A hierarchical organization implies availability of unprecedented mechanisms of compactization, having no remote analogy in techniques, which is best of all traced on the example of DNA compactization.

In nos. 5 and 6 of «Biofizika» 2011, the Editorial Board publishes papers on the materials of the afore- 
mentioned plenary sessions «Biophysics and Nanotechnologies: Problems and Prospects».

The current issue focuses on examples of nanotechnologies in vivo, in the first place on analysis of photosystems, electron transport systems, and the theoretical approaches being developed. Another line is the problems of regulation of gene expression on molecular and cellular levels. Examples of nanotechnologies in medicine, as we hope, will show the role of physical principles in the course of analysis of pathological processes and the role of in vivo nanotechnologies in the development of medical nanotechnologies and material production. Finally, several papers are devoted to the problems of developing the methods of analysis in the physics of single molecules and nanosystems.

The next issue will present the corresponding papers on theoretical research on macromolecules, use of nanoparticles for methodical and medical purposes, and on problems of biosafety.

Chairman of the RAS Scientific Council on Biological Physics A.B. Rubin 